\title{
Constraining X-ray-Induced Photoevaporation of Protoplanetary Disks Orbiting Low-Mass Stars
}

\author{
Kristina M. Punzi ${ }^{1}$, Joel H. Kastner ${ }^{1}$, David Rodriguez ${ }^{2}$, \\ David A. Principe ${ }^{3,4}$, and Laura Vican ${ }^{5}$ \\ ${ }^{1}$ Laboratory for Multiwavelength Astrophysics, Rochester Institute of Technology \\ ${ }^{2}$ Universidad de Chile \\ ${ }^{3}$ Núcleo de Astronomía de la Facultad de Ingeniería, Universidad Diego Portales \\ ${ }^{4}$ Millennium Nucleus Protoplanetary Disks \\ ${ }^{5}$ University of California, Los Angeles
}

\begin{abstract}
Low-mass, pre-main sequence stars possess intense high-energy radiation fields as a result of their strong stellar magnetic activity. This stellar UV and X-ray radiation may have a profound impact on the lifetimes of protoplanetary disks. We aim to constrain the X-rayinduced photoevaporation rates of protoplanetary disks orbiting low-mass stars by analyzing serendipitous XMM-Newton and Chandra X-ray observations of candidate nearby $(\mathrm{D}<100$ pc), young (age $<100 \mathrm{Myr}$ ) M stars identified in the GALEX Nearby Young-Star Survey (GALNYSS).
\end{abstract}

Keywords. stars: low-mass, pre-main sequence; techniques: imaging spectroscopy; X-rays: stars

\section{Introduction}

Low-mass (M-type) stars represent some of the best targets for the discovery of potentially habitable exoplanets due to their low luminosities and the location of their habitable zones. Presently, only a small number of planets have been detected around $\mathrm{M}$ stars, with terrestrial planets being common and giant planets being rare, although these results may be affected by a selection bias (e.g., Mulders et al. 2015, Howard et al. 2012). This trend may be a consequence of the intense high-energy radiation fields of lowmass, pre-main sequence stars. A great deal of mass in protoplanetary disks is lost from the surface of the disk due to heating (photoevaporation) from high-energy radiation from the central star. The X-rays from young stars drive disk dissipation and chemistry, influencing the timescale and conditions for exoplanet formation. According to Owen et al. (2012), stellar X-ray luminosity alone sets the photoevaporation rate. However, Gorti et al. (2015) demonstrate that X-ray spectral hardness is also important. Hence, it is necessary to fully characterize the $\mathrm{X}$-ray radiation fields incident on protoplanetary disks.

\section{Serendipitously Detected X-ray Counterparts}

To constrain the X-ray-induced photoevaporation rates of protoplanetary disks orbiting low-mass stars, we can examine stars from the GALEX Nearby Young-Star Survey (GALNYSS; Rodriguez et al. 2013) that have been serendipitously observed by either XMMNewton or Chandra. GALNYSS combines ultraviolet (GALEX) and near-IR (WISE and 2MASS) photometry with kinematics to identify candidate nearby ( $\mathrm{D}<100 \mathrm{pc}$ ), young (age $<100$ Myr), low-mass (M-type) stars. This survey has identified $>2000$ candidates, with most of the stars having spectral types in the range M3-M4. 


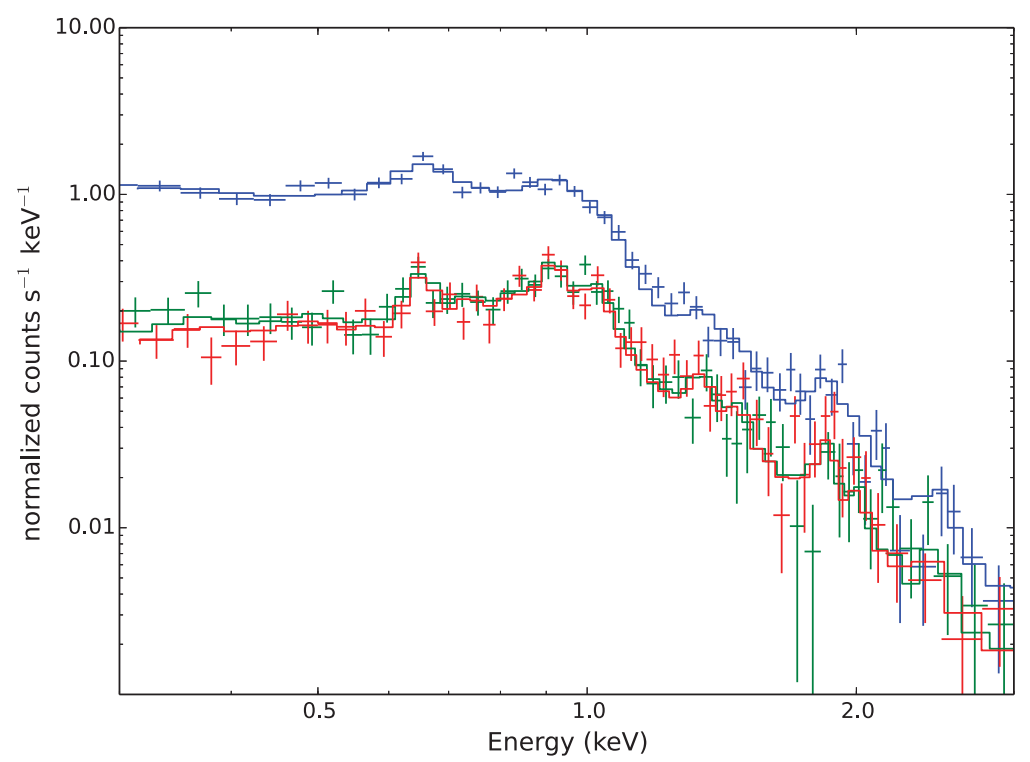

Figure 1. XMM-Newton EPIC extracted spectra (crosses) of J061313.30-274205.6 (spectral type M3, $\beta$ Pictoris Moving Group candidate - 99.24\% likelihood) for pn (blue) and MOS (red and green) detectors. Overplotted are the best-fit models (histograms). Our model predicts plasma temperatures of $\sim 3.6$ and $\sim 12 \mathrm{MK}$ and an X-ray luminosity of $1.2 \times 10^{29} \mathrm{erg} \mathrm{s}^{-1}$ at a distance of $\sim 25 \mathrm{pc}$.

The XMM-Newton data for GALNYSS candidates are being reprocessed and analysed using the Scientific Analysis System following standard procedures $\dagger$. Spectra are extracted from the EPIC detectors using circular regions centered on the 2MASS/WISE positions of the objects. Spectral modelling is performed with XSPEC. An example of the extracted spectra and resulting model fit is displayed in Figure 1. Our models consist of $\mathrm{X}$-ray spectra that result from optically thin plasmas in collisional ionization equilibrium (vapec model in XSPEC $\ddagger$ ). These emission spectra were combined with photoelectric absorption by using the XSPEC model wabs.

\section{Conclusions and Future Work}

Our preliminary results demonstrate that serendipitous XMM-Newton observations of GALNYSS stars are capable of producing useful constraints on the stellar X-ray temperature and luminosity and hence, on photoevaporation due to X-ray irradiation. These early results suggest that X-ray photoevaporation may not account for complete disk dispersal at ages $\sim 8-20$ Myr. For the entire sample of serendipitously observed GALNYSS stars, we will determine X-ray temperatures and luminosities, the potential correlation between $\mathrm{M}$ star $\mathrm{L}_{X}$ and residual disk mass, and the mass dependence of $\mathrm{L}_{X} / \mathrm{L}_{b o l}$.

\section{References}

Gorti, U., Hollenbach, D., \& Dullemond, C. P., 2015, ApJ, 804, 29.

Howard, A. W., Marcy. G. W. Bryson, S. T., et al. 2012, ApJS, 201, 15.

Mulders, G. D., Pascucci, I., \& Apai, D. 2015, ApJ, 798, 112.

Owen, J. E., Clarke, C., J., \& Ercolano, B., 2012, MNRAS, 422, 1880.

Rodriguez, D. R., Zuckerman, B., Kastner, J. H., et al., 2013, ApJ, 744, 101.

$\dagger$ See the SAS documentation at http://xmm.esac.esa.int/sas/current/documentation/threads.

$\ddagger$ See the XSPEC documentation at https://heasarc.gsfc.nasa.gov/xanadu/xspec/manual/Models.html for a description of the models. 